\title{
CONSTRUCTION AND QUALITATIVE BEHAVIOR OF SOLUTIONS FOR A SYSTEM OF NONLINEAR HYPERBOLIC CONSERVATION LAWS WITH DAMPING
}

\author{
BY
}

\section{HSIAO AND S. Q. TANG}

Academia Sinica, Institute of Mathematics, Beijing 100080, China

1. Introduction. Consider the following hyperbolic conservation laws with damping:

$$
\begin{gathered}
v_{t}-u_{x}=0 \\
u_{t}+p(v)_{x}=-\alpha u, \quad \alpha>0, \quad p^{\prime}(v)<0 .
\end{gathered}
$$

This system may be viewed as isentropic Euler equations in Lagrangian coordinates with friction term, $-\alpha u$, for the momentum equation that models the compressible flow through porous media where $u$ is the velocity, $v>0$ is the specific volume, and $p(v)$ is the pressure.

The well-known porous media equation can be obtained by approximating the second equation with Darcy's law

$$
\begin{gathered}
v_{t}=\frac{-1}{\alpha} p(v)_{x x}, \\
p(v)_{x}=-\alpha u .
\end{gathered}
$$

It is proved in [HL] that Darcy's law can be obtained, in fact, from the more complete equation (1.1) time-asymptotically. Namely, solutions of (1.1) tend to those of (1.2) as the time $t$ tends to infinity. In other words, nonlinear diffusive phenomena in the solutions of (1.1) are discovered in [HL] where the diffusion effect created by the damping is studied for a solution without shock waves. In the case when shock waves may develop in the solution of (1.1) with rough data, it is expected that (1.2) would still model the time-asymptotic behavior of (1.1).

As a part of the program to prove this expectation we first study the discontinuous initial value problem of (1.1), namely

$$
\left(u_{0}(x), v_{0}(x)\right)= \begin{cases}\left(u_{-}(x), v_{-}(x)\right) & \text { for } x<0, \\ \left(u_{+}(x), v_{+}(x)\right) & \text { for } x>0\end{cases}
$$

Received December 14, 1992.

1991 Mathematics Subject Classification. Primary 35, 76.

Project supported by the National Natural Science Foundation of China. 
where $u_{\mp}(x), v_{\mp}(x)$ are smooth functions such that $\lim _{x \rightarrow 0}\left(u_{\mp}(x), v_{\mp}(x)\right)=$ $\left(u^{\mp}, v^{\mp}\right)$ with $v^{\mp}>0$. This kind of discontinuous initial value problem is significant in the qualitative theory of solutions for inhomogeneous hyperbolic systems and in the applications as well.

For the system without the damping term, i.e.,

$$
\begin{gathered}
v_{t}-u_{x}=0, \\
u_{t}+p\left(v_{x}\right)=0, \quad p^{\prime}(v)<0,
\end{gathered}
$$

the same kind of discontinuous initial value problem (1.3), (1.4) has been studied in [LZ] where $u_{\mp}(x), v_{\mp}(x)$ are considered as perturbed Riemann data. It has been proved under certain restrictions on the initial data (1.3) that the problem (1.4), (1.3) admits a unique global solution in a class of piecewise-continuous and piecewise-smooth functions and the solution has a global structure similar to that of the corresponding Riemann problem with Riemann data $\left(u^{-}, v^{-}\right)$and $\left(u^{+}, v^{+}\right)$. The Riemann problem for (1.4) has been studied very well (see [CH], [LI $\left.{ }_{1}\right],[D D]$, [SM], etc.).

For the system (1.1) with damping term, however, the Riemann problem is much more complicated since there is no self-similar solution anymore, and it has not been well studied in the literature.

We investigate the perturbed Riemann problem (1.1), (1.3) and construct the globally-defined piecewise-continuous and piecewise-smooth solutions with showing the qualitative behavior in separate papers, for convenience, corresponding to different relative locations of the two states $\left(u^{-}, v^{-}\right)$and $\left(u^{+}, v^{+}\right)$in the phase plane. For simplicity, we take a typical form of the state function $p(v)$ to discuss, instead of a general form, namely $p(v)=a v^{-\gamma}$ with $a>0$ and $1 \leq \gamma<3$, which is the state function for polytropic gas. The present paper and the coming paper [HT] deal with the case in which the two states $\left(u^{-}, v^{-}\right)$and $\left(u^{+}, v^{+}\right)$are connected by a backward shock curve and a forward shock curve subsequently in the phase plane. This paper handles the case when $\gamma$ is 1 or nearly 1 ; the investigation for the case $1<\gamma<3$ is in [HT]. For other kinds of relations between $\left(u^{-}, v^{-}\right)$and $\left(u^{+}, v^{+}\right)$, the result can be found in $\left[\mathrm{TX}_{1}\right]$ and $\left[\mathrm{TX}_{2}\right]$.

Under certain restrictions on the perturbation in $\left(u_{-}(x), v_{-}(x)\right)$ and $\left(u_{+}(x)\right.$, $\left.v_{+}(x)\right)$, we prove that the problem (1.1), (1.3) admits a unique global discontinuous solution on $t \geq 0$ in a class of piecewise-continuous and piecewise-smooth functions which only contain two entropy shocks-a backward shock and a forward shock. The shocks do not disappear for any finite time and do disappear with the strength decay exponentially fast when time $t$ tends to infinity. We first prove the result for unperturbed Riemann data with $\gamma=1$ in Sec. 2. The result for perturbed Riemann data with $\gamma=1$ is obtained in Sec. 3. The last section concerns the case when $\gamma$ is nearly 1 .

For the general Cauchy problem of inhomogeneous hyperbolic systems, the discussion on the existence of weak solutions can also be found in $[\mathrm{DH}],\left[\mathrm{LI}_{2}\right],[\mathrm{YW}]$, [DLC], [HM], [LT], etc. 
2. Construction and behavior of the solution to the Riemann problem. We consider the unperturbed initial data (1.3) in this section, namely,

$$
\left(u_{0}(x), v_{0}(x)\right)= \begin{cases}\left(u^{-}, v^{-}\right) & x<0, \\ \left(u^{+}, v^{+}\right) & x>0,\end{cases}
$$

which is called Riemann data in the theory for systems of hyperbolic conservation laws.

Consider the system (1.1) where $p(v)=a / v$ in this section. Without loss of generality, we take $a=1$ and $\alpha=1$ in (1.1) for convenience. Thus, the characteristic speed takes the form $\lambda=-1 / v$ and $\mu=1 / v$, respectively, corresponding to the backward and forward family.

Introduce Riemann invariants

$$
\begin{aligned}
& r=u-\varphi, \\
& s=u+\varphi
\end{aligned}
$$

where $\varphi$ is defined by $\varphi=-\ln v$. Then the system (1.1) can be written as

$$
\begin{aligned}
& r_{t}+\lambda r_{x}=-\frac{1}{2}(r+s), \\
& s_{t}+\mu s_{x}=-\frac{1}{2}(r+s),
\end{aligned}
$$

which is equivalent to (1.1) wherever the solution is smooth. We may use the following notation:

$$
\begin{aligned}
& { }^{\prime}=\frac{\partial}{\partial t}+\lambda \frac{\partial}{\partial x}, \\
& { }^{\prime}=\frac{\partial}{\partial t}+\mu \frac{\partial}{\partial x}
\end{aligned}
$$

in which (2.4) turns into

$$
\begin{aligned}
& r^{\prime}=-\frac{1}{2}(r+s), \\
& s^{\prime}=-\frac{1}{2}(r+s) .
\end{aligned}
$$

Turn to a discontinuous solution of (1.1) next. A discontinuity in a solution $(u, v)$ of $(1.1)$ at $(t, x(t))$ should satisfy the Rankine-Hugoniot compatibility condition which implies that either

or

$$
\left\{\begin{array}{l}
\frac{d x(t)}{d t}=\frac{-1}{\sqrt{v_{R} v_{L}}}, \\
\frac{u_{R}-u_{L}}{v_{R}-v_{L}}=\frac{1}{\sqrt{v_{R} v_{L}}},
\end{array}\right.
$$

$$
\left\{\begin{array}{l}
\frac{d x(t)}{d t}=\frac{1}{\sqrt{v_{R} v_{L}}}, \\
\frac{u_{R}-u_{L}}{v_{R}-v_{L}}=-\frac{1}{\sqrt{v_{R} v_{L}}},
\end{array}\right.
$$

respectively, where $\left(u_{R}, v_{R}\right)=(u, v)(t, x(t)+0),\left(u_{L}, v_{L}\right)=(u, v)(t, x(t)-0)$. A discontinuity is called a backward shock (or forward shock) if (2.7) (or (2.8)) holds and the entropy condition is satisfied as well, namely,

$$
v_{R}<v_{L}
$$

$$
v_{R}>v_{L} \text {. }
$$


By using (2.2) and (2.3), it is easy to write (2.7) and (2.8) in $(r, s)$ variables. Corresponding to (2.7),

$$
\left\{\begin{array}{l}
\frac{d x(t)}{d t}=f_{1}\left(s_{R}-r_{R}, s_{L}-r_{L}\right), \\
s_{R}-s_{L}=h_{1}\left(r_{R}-r_{L}\right)
\end{array}\right.
$$

holds while (2.8) corresponds to

$$
\left\{\begin{array}{l}
\frac{d x(t)}{d t}=f_{2}\left(s_{R}-r_{R}, s_{L}-r_{L}\right), \\
r_{R}-r_{L}=h_{2}\left(s_{R}-s_{L}\right)
\end{array}\right.
$$

where $h_{1}\left(r_{R}-r_{L}\right)$ is determined by $H\left(s_{R}-s_{L}, r_{R}-r_{L}\right)=0, h_{2}\left(s_{R}-s_{L}\right)$ is determined by $H\left(r_{R}-r_{L}, s_{R}-s_{L}\right)=0$, and $H(\xi, \eta)=\frac{\xi+\eta}{2}+e^{(\xi-\eta) / 4}-e^{(\eta-\xi) / 4}$, $f_{1}=-e^{\left(\left(s_{R}-r_{R}\right)+\left(s_{L}-r_{L}\right)\right) / 4}=-f_{2}$.

Furthermore, the entropy condition (2.9) (or (2.10)) can be expressed in $(r, s)$ variables as

(or

$$
\begin{aligned}
& s_{R}-r_{R}>s_{L}-r_{L} \\
& s_{R}-r_{R}<s_{L}-r_{L} .
\end{aligned}
$$

We may choose either $(u, v)$ or $(r, s)$ to use in the coming discussion according to convenience.

The initial data (2.1) satisfies the following condition in the present paper. There exists a state $\left(r_{0}, s_{0}\right)\left(\right.$ or $\left(u_{0}, v_{0}\right)$ in $(u, v)$ variables) such that

$$
\left\{\begin{array}{l}
s_{0}-s^{-}=h_{1}\left(r_{0}-r^{-}\right), \\
r^{+}-r_{0}=h_{2}\left(s^{+}-s_{0}\right), \text { and } \\
s_{0}-r_{0}>s^{-}-r^{-}, \\
s_{0}-r_{0}>s^{+}-r^{+}
\end{array}\right.
$$

where $\left(r^{+}, s^{+}\right)$and $\left(r^{-}, s^{-}\right)$are determined by $\left(u^{+}, v^{+}\right)$and $\left(u^{-}, v^{-}\right)$through (2.2) and (2.3). This means that the two states $\left(u^{-}, v^{-}\right)$and $\left(u^{+}, v^{+}\right)$are connected by a backward shock curve and a forward shock curve subsequently in the phase plane.

We construct the global discontinuous solution for (1.1), (2.1), (2.15) next.

First of all, by using the initial data on $x \geq 0$, we solve the corresponding initial value problem for (1.1). It is easy to know that this problem admits a unique global $C^{1}$ solution $\left(r_{+}(t, x), s_{+}(x, t)\right)$ on the domain $\widehat{R}_{+}=\{(t, x) \mid t \geq 0, x \geq$ $\left.\xi_{+}(t), d \xi_{+}(t) / d t=\mu\left(v_{+}\left(t, \xi_{+}(t)\right)\right)\right\}$. Similarly, we solve the corresponding initial value problem for (1.1) by means of the initial data on $x \leq 0$ and get a unique global $C^{1}$ solution $\left(r_{-}(t, x), s_{-}(t, x)\right)$ on the domain $\widehat{R}_{-}=\{(t, x) \mid t \geq 0, x \leq$ $\left.\xi_{-}(t), d \xi_{-}(t) / d t=\lambda\left(v_{-}\left(t, \xi_{-}(t)\right)\right)\right\}$.

By the local existence theorem in [LY], this discontinuous initial value problem (1.1), (2.1) admits a unique discontinuous solution at least on a local domain $R(\delta)=$ $\{(t, x) \mid 0 \leq t \leq \delta,-\infty<x<\infty\}$ in a class of piecewise-smooth functions and this solution contains only a backward shock $x=x_{1}(t)$ and a forward shock $x=$ $x_{2}(t)$ passing through the origin. In view of the entropy condition on the initial discontinuities, it is known that $x=x_{1}(t)$ must be located on the left side of $x=$ $\xi_{-}(t)$ while $x=x_{2}(t)$ is on the right side of both $x=\xi_{+}(t)$ and $x=x_{1}(t)$. 
Therefore, the solution on the left side of $x=x_{1}(t)$ and on the right side of $x=x_{2}(t)$ will be furnished by $\left(r_{-}(t, x), s_{-}(t, x)\right)$ and $\left(r_{+}(t, x), s_{+}(t, x)\right)$, respectively, and one is required to solve the following free boundary problem on the angular domain $R=\left\{(t, x) \mid t \geq 0, x_{1}(t) \leq x \leq x_{2}(t)\right\}$ for (1.1) in order to construct the globallydefined discontinuous solution for (1.1), (2.1) that contains only two shocks.

(FBP): On the free boundary $x=x_{1}(t) \quad\left(x_{1}(0)=0\right)$

$$
\begin{gathered}
s\left(t, x_{1}(t)\right)=s_{-}\left(t, x_{1}(t)\right)+h_{1}\left(r\left(t, x_{1}(t)\right)-r_{-}\left(t, x_{1}(t)\right)\right) \\
\frac{d x_{1}(t)}{d t}=f_{1}\left(s\left(t, x_{1}(t)\right)-r\left(t, x_{1}(t)\right), s_{-}\left(t, x_{1}(t)\right)-r_{-}\left(t, x_{1}(t)\right)\right) .
\end{gathered}
$$

On the free boundary $x=x_{2}(t) \quad\left(x_{2}(0)=0\right)$

$$
\begin{gathered}
r\left(t, x_{2}(t)\right)=r_{+}\left(t, x_{2}(t)\right)-h_{2}\left(s_{+}\left(t, x_{2}(t)\right)-s\left(t, x_{2}(t)\right)\right) \\
\frac{d x_{2}(t)}{d t}=f_{2}\left(s_{+}\left(t, x_{2}(t)\right)-r_{+}\left(t, x_{2}(t)\right), s\left(t, x_{2}(t)\right)-r\left(t, x_{2}(t)\right)\right)
\end{gathered}
$$

where $\left(f_{1}, h_{1}\right)$ and $\left(f_{2}, h_{2}\right)$ are defined in (2.11) and (2.12), respectively. Moreover, the entropy condition has to be satisfied on the free boundaries, namely,

$$
\begin{array}{ll}
\text { on } x=x_{1}(t), & (s-r)\left(t, x_{1}(t)\right)>\left(s_{-}-r_{-}\right)\left(t, x_{1}(t)\right), \\
\text { on } x=x_{2}(t), & (s-r)\left(t, x_{2}(t)\right)>\left(s_{+}-r_{+}\right)\left(t, x_{2}(t)\right) .
\end{array}
$$

We prove the main theorem next. Without loss of generality, we assume that

$$
u^{ \pm}>0 \text { and } 0<v^{ \pm}<1 \text {; }
$$

moreover, $v^{+} \geq v^{-}$.

The other cases can be discussed similarly.

Condition A. $\sqrt{v^{+}} / \sqrt{v_{0}}<\left(2^{1 / 3}+1\right) /\left(2^{1 / 3}-1\right)$ where $v_{0}$ is given in $(2.15)$.

Theorem 2.1. Under Condition A, the above free boundary problem (1.1), (2.16), (2.17) admits a global classical solution $(r, s) \in C^{1}$ in $R$ with $x_{i}(t) \in C^{2} \quad(i=1,2)$ on which (2.18) holds. Furthermore, this solution possesses the following properties. For any $(t, x) \in R$,

$$
\begin{aligned}
& s_{*} \leq s(t, x) \leq s^{-}, \quad-s^{-} \leq r(t, x) \leq r_{0}, \quad v(t, x) \leq \max \left\{v^{-}, v^{+}\right\}=v^{+}, \\
& \min \left\{\min _{\substack{x_{2}(\tau ; t, x) \\
\alpha \leq \tau \leq t}} v^{1 / 2},\left(v^{-1 / 2} s_{x}+v^{1 / 2}\right)\left(\alpha, x_{1}(\alpha)\right)\right\} \\
& \quad \leq v^{-1 / 2} s_{x}+v^{1 / 2} \leq \max \left\{\max _{\substack{x_{2}(\tau ; t, x) \\
\alpha \leq \tau \leq t}} v^{1 / 2},\left(v^{-1 / 2} s_{x}+v^{1 / 2}\right)\left(\alpha, x_{1}(\alpha)\right)\right\}, \\
& \min \left\{\min _{\substack{x_{1}(\tau ; t, x) \\
\beta \leq \tau \leq t}} v^{1 / 2},\left(v^{-1 / 2} r_{x}+v^{1 / 2}\right)\left(\beta, x_{2}(\beta)\right)\right\} \\
& \quad \leq v^{-1 / 2} r_{x}+v^{1 / 2} \leq \max \left\{\max _{\substack{x_{1}(\tau ; t, x) \\
\beta \leq \tau \leq t}} v^{1 / 2}\left(v^{-1 / 2} r_{x}+v^{1 / 2}\right)\left(\beta, x_{2}(\beta)\right)\right\}
\end{aligned}
$$


where $x=x_{1}(\tau, t, x)$ denotes the backward characteristic that meets $x=x_{2}(t)$ at $\left(\beta, x_{2}(\beta)\right)$ when $\tau=\beta$, and $x=x_{2}(\tau ; t, x)$ denotes the forward characteristic that meets $x=x_{1}(t)$ at $\left(\alpha, x_{1}(\alpha)\right)$ when $\tau=\alpha, s_{*}=\left(s^{+}-r^{+}\right) / 2$.

Moreover, for any point $\left(t, x_{1}(t)\right), 0 \leq t<\infty$,

$$
0<v^{-1 / 2} s_{x}+v^{1 / 2}<\left(v^{-}\right)^{1 / 2}
$$

and

$$
\left(\sqrt{v^{-}}-\sqrt{v_{0}}\right) e^{-A_{3} t} \leq \sqrt{v_{-}}-\sqrt{v} \leq\left(\sqrt{v^{-}}-\sqrt{v_{0}}\right) e^{-A_{4} t}
$$

hold; for any point $\left(t, x_{2}(t)\right), 0 \leq t<\infty$,

$$
0<v^{-1 / 2} r_{x}+v^{1 / 2}<\left(v^{+}\right)^{1 / 2}
$$

and

$$
\left(\sqrt{v^{+}}-\sqrt{v_{0}}\right) e^{-A_{1} t} \leq \sqrt{v_{+}}-\sqrt{v} \leq\left(\sqrt{v^{+}}-\sqrt{v_{0}}\right) e^{-A_{2} t}
$$

hold, where $v_{-}=v_{-}\left(t, x_{1}(t)\right), v_{+}=v_{+}\left(t, x_{2}(t)\right)$, and $A_{i}$ is a positive constant $(i=1,2,3,4)$. In this section it is easy to show that $v_{-}=v^{-}$and $v_{+}=v^{+}$.

RemarK 2.2. Let $\xi_{0}>0$ be the smallest root of the equation $\xi^{3}-7 \xi^{2}-\xi-1=0$. If we assume that $\sqrt{v_{+}}<\xi_{0} \sqrt{v_{-}}$and $\left(\sqrt{v_{-}}+\sqrt{v_{0}}\right)^{3}-\left(\sqrt{v_{-}}+\sqrt{v_{0}}\right)^{2}\left(\sqrt{v_{-}}+\sqrt{v_{+}}\right) \geq 0$, then we get more estimates on $x=x_{i}(t) \quad(i=1,2)$, namely, $v^{-1 / 2} s_{x}+v^{1 / 2} \geq v_{0}^{1 / 2}$ on $x=x_{1}(t)$ and $v^{-1 / 2} r_{x}+v^{1 / 2} \geq v_{0}^{1 / 2}$ on $x=x_{2}(t)$, respectively. These estimates imply an additional estimate $v \geq v_{0}$ in the whole domain $R$. The above assumptions can be guaranteed, in fact, if one changes the number on the right side of Condition A into a smaller number, say, any number that is less than or equal to 7.15.

For proving this theorem, we need the following lemmas.

Lemma 2.3. Suppose that the classical solution of (1.1), (2.16), (2.17) exists in $R(T)$ : $\left\{(t, x) \mid 0 \leq t \leq T, x_{1}(t) \leq x \leq x_{2}(t)\right\}$. Then

$$
\begin{gathered}
s_{x}=\frac{4 v \sqrt{v_{-}}\left(\sqrt{v_{-}}-\sqrt{v}\right)}{\left(\sqrt{v_{-}}+\sqrt{v}\right)^{2}}-\left(\frac{\sqrt{v_{-}}-\sqrt{v}}{\sqrt{v_{-}}+\sqrt{v}}\right)^{3} r_{x} \\
v^{-1 / 2} s_{x}+v^{1 / 2}=\sqrt{v_{-}}\left[1-\left(\frac{\sqrt{v_{-}}-\sqrt{v}}{\sqrt{v_{-}}+\sqrt{v}}\right)^{3}\right]-\left(\frac{\sqrt{v_{-}}-\sqrt{v}}{\sqrt{v_{-}}+\sqrt{v}}\right)^{3}\left(v^{-1 / 2} r_{x}+v^{1 / 2}\right)
\end{gathered}
$$

hold along $x=x_{1}(t)$ while

$$
\begin{gathered}
r_{x}=\frac{4 v \sqrt{v_{+}}\left(\sqrt{v_{+}}-\sqrt{v}\right)}{\left(\sqrt{v_{+}}+\sqrt{v}\right)^{3}}-\left(\frac{\sqrt{v_{+}}-\sqrt{v}}{\sqrt{v_{+}+\sqrt{v}}}\right)^{3} s_{x} \\
v^{-1 / 2} r_{x}+v^{1 / 2}=\sqrt{v_{+}}\left[1-\left(\frac{\sqrt{v_{+}}-\sqrt{v}}{\sqrt{v_{+}}+\sqrt{v}}\right)^{3}\right]-\left(\frac{\sqrt{v_{+}}-\sqrt{v}}{\sqrt{v_{+}}+\sqrt{v}}\right)^{3}\left(v^{-1 / 2} s_{x}+v^{1 / 2}\right)
\end{gathered}
$$

hold along $x=x_{2}(t)$. 
Proof. Due to $(2.7)_{2},(2.2)$, and (2.3),

$\left[r\left(t, x_{1}(t)\right)+s\left(t, x_{1}(t)\right)\right]-\left[r_{-}\left(t, x_{1}(t)\right)+s_{-}\left(t, x_{1}(t)\right)\right]=2 \cdot \frac{\left[v\left(t, x_{1}(t)\right)-v_{-}\left(t, x_{1}(t)\right)\right]}{\sqrt{v\left(t, x_{1}(t)\right) \cdot v_{-}\left(t, x_{1}(t)\right)}}$

and

$$
\frac{d x_{1}(t)}{d t}=-\frac{1}{\sqrt{v\left(t, x_{1}(t)\right) \cdot v_{-}\left(t, x_{1}(t)\right)}}
$$

hold on $x=x_{1}(t)$.

Differentiating (2.30) with respect to $t$, we obtain (2.26) with the help of (2.31), (2.2)-(2.4), and the conditions on $\left(r_{-}(t, x), s_{-}(t, x)\right)$. (2.28) can be obtained in a similar way.

Let us denote the following statements by $\left(\mathrm{H}_{1}\right)$ and $\left(\mathrm{H}_{2}\right)$ respectively.

$$
(r, s) \in C^{1} \text { on } x_{i}(t) \text { and } x_{i}(t) \in C^{2} \quad(i=1,2)(t \geq 0) .
$$

$\left(\mathrm{H}_{2}\right)$ Along $x=x_{1}(t), t \geq 0,0<v^{-1 / 2} s_{x}+v^{1 / 2}<\left(v^{-}\right)^{1 / 2}$ and $\frac{-1}{\left(\sqrt{v_{-}}-\sqrt{v}\right)} \cdot \frac{d\left(\sqrt{v_{-}}-\sqrt{v}\right)}{d t}$ $\in\left[A_{4}, A_{3}\right]$ hold for positive constants $A_{3}>A_{4}>0$.

Along $x=x_{2}(t), t \geq 0,0<v^{-1 / 2} r_{x}+v^{1 / 2}<\left(v^{+}\right)^{1 / 2}$ and $\frac{-1}{\left(\sqrt{v_{+}}-\sqrt{v}\right)} \frac{d\left(\sqrt{v_{+}}-\sqrt{v}\right)}{d t} \in$ [ $A_{2}, A_{1}$ ] hold for positive constants $A_{1}>A_{2}>0$.

REMARK 2.4. $\left(\mathrm{H}_{2}\right)$ implies that $(s-r)\left(\alpha, x_{1}(\alpha)\right)>\left(s_{-}-r_{-}\right)\left(\alpha, x_{1}(\alpha)\right)$ and that $(s-r)\left(\beta, x_{2}(\beta)\right)>\left(s_{+}-r_{+}\right)\left(\beta, x_{2}(\beta)\right)$ for $\alpha \geq 0$ and $\beta \geq 0$, respectively.

Lemma 2.5. The classical solution defined in $R(T)$ satisfies the estimates cited in (2.20), (2.21), (2.23), and (2.25) if $\left(\mathrm{H}_{1}\right)$ and $\left(\mathrm{H}_{2}\right)$ hold for $t \in[0, T]$.

Proof. Due to the characteristic equations (2.4), it follows that

$$
\begin{aligned}
& r(t, x)=e^{(\beta-t) / 2} r\left(\beta, x_{2}(\beta)\right)-\int_{\beta}^{t} \frac{1}{2} s\left(\tau ; x_{1}(\tau ; t, x)\right) e^{(\tau-t) / 2} d \tau \\
& s(t, x)=e^{(\alpha-t) / 2} s\left(\alpha, x_{1}(\alpha)\right)-\int_{\alpha}^{t} \frac{1}{2} r\left(\tau ; x_{2}(\tau ; t, x)\right) e^{(\tau-t) / 2} d \tau
\end{aligned}
$$

for any $(t, x) \in R(T)$, where $x_{1}(\tau ; t, x)$ and $x_{2}(\tau ; t, x)$ are defined as in Theorem 2.1 , and $\alpha$ and $\beta$ have the same sense as in Theorem 2.1 as well.

Denote $M=\max _{R(T)}\{|r|,|s|\}$. If $M$ is reached at some $(t, x) \in R(T)$ with $|r(t, x)|$, it follows that

$$
M \leq e^{(\beta-t) / 2}\left|r\left(\beta, x_{2}(\beta)\right)\right|+\int_{\beta}^{t} \frac{1}{2} M e^{(\tau-t) / 2} d \tau,
$$

which implies that $M \leq\left|r\left(\beta, x_{2}(\beta)\right)\right|$. Namely, either $M \leq r\left(\beta, x_{2}(\beta)\right)$ if $r\left(\beta, x_{2}(\beta)\right) \geq 0$, or $M \leq-r\left(\beta, x_{2}(\beta)\right)$ if $r\left(\beta, x_{2}(\beta)\right)<0$. However, the first inequality gives a contradiction since $r\left(\beta, x_{2}(\beta)\right)<s\left(\beta, x_{2}(\beta)\right)$ (due to Remark 2.4 and (2.19)) while the second inequality implies a contradiction too. In fact, it is easy to see that $\inf _{t \geq 0} r_{+}\left(t, x_{2}(t)\right)=r_{*}$, where $r_{*}=-s_{*}$. Remark 2.4 shows that $r\left(t, x_{2}(t)\right)>r_{+}\left(t, x_{2}(t)\right)$. Thus, it follows that $M \leq-r_{*}=s_{*}<s^{-}$, a contradiction. 
Thus, $M$ must be reached at some $(t, x) \in R(T)$ with $|s(t, x)|$. Similarly to the above argument, it follows that

$$
M \leq\left|s\left(\alpha, x_{1}(\alpha)\right)\right|=s\left(\alpha, x_{1}(\alpha)\right) .
$$

This shows that $M \leq s^{-}$.

Let

$$
\begin{aligned}
f & =\frac{1}{2} v^{-1 / 2}, & & g=2 v^{1 / 2}=1 / f, \\
\theta & =v^{-1 / 2} r_{x}, & & =v^{-1 / 2} s_{x}, \\
w & =\theta+\frac{1}{2} g, & & y=\varphi+\frac{1}{2} g .
\end{aligned}
$$

It is easy to show that

$$
\begin{aligned}
w^{\prime} & =w\left(\frac{1}{2}-f w\right), \\
y^{\prime} & =y\left(\frac{1}{2}-f y\right),
\end{aligned}
$$

by which the estimates (2.21) can be obtained with the help of $\left(\mathrm{H}_{1}\right)$ and $\left(\mathrm{H}_{2}\right)$.

We finish the estimate (2.20) next. Let $N(T)=\max _{R(T)}\{\sqrt{v}\}$ being reached at $(t, x) \in R(T)$.

Since $(\sqrt{v})^{\prime}=\frac{1}{2}\left(\frac{s_{x}}{\sqrt{v}}+\sqrt{v}\right)-\frac{1}{2} \sqrt{v}$, it can be shown that

$$
\begin{aligned}
N(T) & =\sqrt{v(t, x)}=\sqrt{v\left(\beta, x_{2}(\beta)\right)} e^{(\beta-t) / 2}+\frac{1}{2} \int_{\beta}^{t}\left(\frac{s_{x}}{\sqrt{v}}+\sqrt{v}\right) e^{(\tau-t) / 2} d \tau \\
& \leq \sqrt{v\left(\beta, x_{2}(\beta)\right)} e^{(\beta-t) / 2}+\left(1-e^{(\beta-t) / 2}\right) \max \left\{N(T), \sqrt{v^{-}}\right\}
\end{aligned}
$$

due to $(2.21)$ and $H_{2}$. Thus, $N(T) \leq \max \left\{\sqrt{v^{+}}, \sqrt{v^{-}}\right\}$in view of Remark 2.4, namely, $v \leq \max \left\{v^{+}, v^{-}\right\}=v^{+}$.

Furthermore, it is clear that the region $s<s_{*}$ cannot be reached, due to $(2.4)_{2}$ and the fact that $v \leq v^{+}$. Hence, $s_{*} \leq s \leq s^{-}$follows. This fact combined with (2.32) and $\mathrm{H}_{2}$ together, implies that $r(t, x) \leq r_{0}$. The proof of Lemma 2.5 is then finished.

By using Lemma 2.3, it is easy to prove that

LEMMA 2.6. Suppose that the classical solution of (1.1), (2.16), (2.17) exists in $R(T)$. Then

$$
\frac{-1}{\left(\sqrt{v_{-}}-\sqrt{v}\right)} \frac{d\left(\sqrt{v_{-}}-\sqrt{v}\right)}{d t}=\frac{\sqrt{v}}{\left(\sqrt{v_{-}}+\sqrt{v}\right)^{2}}\left(v^{-1 / 2} r_{x}+v^{1 / 2}\right)+\frac{\sqrt{v v_{-}}}{\left(\sqrt{v_{-}}+\sqrt{v}\right)^{2}}
$$

holds along $x=x_{1}(t)$ while

$$
\frac{-1}{\left(\sqrt{v_{+}}-\sqrt{v}\right)} \frac{d\left(\sqrt{v_{+}}-\sqrt{v}\right)}{d t}=\frac{\sqrt{v}}{\left(\sqrt{v_{+}}+\sqrt{v}\right)^{2}}\left(v^{-1 / 2} s_{x}+v^{1 / 2}\right)+\frac{\sqrt{v v_{+}}}{\left(\sqrt{v_{+}}+\sqrt{v}\right)^{2}}
$$

holds along $x=x_{2}(t)$. 
Lemma 2.7. There exists a small constant $\delta_{0}$ such that the solution of (1.1), (2.16), (2.17) exists in $R\left(\delta_{0}\right)$ for which $\left(\mathrm{H}_{1}\right)$ and $\left(\mathrm{H}_{2}\right)$ hold.

Proof. The existence follows from the local existence as mentioned before and it is obvious how to verify $\left(\mathbf{H}_{1}\right)$. Moreover, it can be shown that $v \in\left[v_{0}\left(1-\bar{\delta}_{0}\right)\right.$, $\left.v_{0}(1+\bar{\delta})\right]$ for some small positive constant $\bar{\delta}_{0}\left(0<\bar{\delta}_{0}<1\right)$ if $\delta_{0}$ is small enough.

Due to Lemma 2.3, it can be claimed that

$$
\begin{aligned}
& \lim _{t \rightarrow 0}\left(v^{-1 / 2} s_{x}+v^{1 / 2}\right)\left(t, x_{1}(t)\right)= \\
& \frac{2 v_{0}\left(\sqrt{v^{+}}+\sqrt{v^{-}}\right)\left[v_{0}\left(3 v^{+}+3 v^{-}+v_{0}\right)+v^{+} v^{-}+8 v^{+} \sqrt{v^{-} v_{0}}+8 \sqrt{v^{+} v^{-}}\left(\sqrt{v^{+}}-\sqrt{v_{0}}\right)\right]}{\left(\sqrt{v^{-}}+\sqrt{v_{0}}\right)^{3}\left(\sqrt{v^{+}}+\sqrt{v_{0}}\right)^{3}-\left(\sqrt{v^{-}}-\sqrt{v_{0}}\right)^{3}\left(\sqrt{v^{+}}-\sqrt{v_{0}}\right)^{3}}>0
\end{aligned}
$$

and

$$
\begin{aligned}
& \lim _{t \rightarrow 0}\left(v^{-1 / 2} r_{x}+v^{1 / 2}\right)\left(t, x_{2}(t)\right)= \\
& \frac{2 v_{0}\left(\sqrt{v^{+}}+\sqrt{v^{-}}\right)\left[v_{0}\left(3 v^{+}+3 v^{-}+v_{0}\right)+v^{+} v^{-}+8 v^{-} \sqrt{v^{+} v_{0}}+8 \sqrt{v^{-}} v^{+}\left(\sqrt{v^{-}}-\sqrt{v_{0}}\right)\right]}{\left(\sqrt{v^{-}}+\sqrt{v_{0}}\right)^{3}\left(\sqrt{v^{+}}+\sqrt{v_{0}}\right)^{3}-\left(\sqrt{v^{-}}-\sqrt{v_{0}}\right)^{3}\left(\sqrt{v^{+}}-\sqrt{v_{0}}\right)^{3}}>0 .
\end{aligned}
$$

On the other hand, it can easily be obtained, by throwing off negative items, that

$$
\lim _{t \rightarrow 0}\left(v^{-1 / 2} s_{x}+v^{1 / 2}\right)\left(t, x_{1}(t)\right)<\sqrt{v^{-}}
$$

and

$$
\lim _{t \rightarrow 0}\left(v^{-1 / 2} r_{x}+v^{1 / 2}\right)\left(t, x_{2}(t)\right)<\sqrt{v^{+}} .
$$

In view of the above estimates and Lemma 2.6, we are able to find constants $A_{3}>$ $A_{4}>0, A_{1}>A_{2}>0$, such that

$$
\frac{-1}{\sqrt{v_{-}}-\sqrt{v}} \frac{d\left(\sqrt{v_{-}}-\sqrt{v}\right)}{d t} \in\left[A_{4}, A_{3}\right] \text { along } x=x_{1}(t)
$$

and

$$
\frac{-1}{\sqrt{v_{+}}-\sqrt{v}} \frac{d\left(\sqrt{v_{+}}-\sqrt{v}\right)}{d t} \in\left[A_{2}, A_{1}\right] \text { along } x=x_{2}(t) \text {. }
$$

LemMA 2.8. Under Condition A, the solution constructed by extension reserves $\left(\mathrm{H}_{1}\right)$ and $\left(\mathrm{H}_{2}\right)$ in each step. Namely, if $\left(\mathrm{H}_{1}\right)$ and $\left(\mathrm{H}_{2}\right)$ hold in $R\left(T_{0}\right)$ where the classical solution of (1.1), (2.16), (2.17) is defined, then the solution defined in $R\left(T_{0}+\delta\right)$ still satisfies $\left(\mathrm{H}_{1}\right)$ and $\left(\mathrm{H}_{2}\right)$, provided $\delta$ is small enough.

Proof. Denote $T^{*}=T_{0}+\delta$. It is easy for $\left(\mathrm{H}_{1}\right)$ to be verified. We verify $\left(\mathrm{H}_{2}\right)$ next. It is not difficult to see that there is a constant $0<\varepsilon<1$ such that $v \in$ $\left[\hat{v}(1-\varepsilon), v^{+}(1+\varepsilon)\right]$ in $R\left(T^{*}\right)$ since $\delta_{0}$ is small enough and $\left(\mathrm{H}_{2}\right)$ holds in $R\left(T_{0}\right)$, where $\hat{v}=e^{-s^{-}}$. For the same reason, the strength of discontinuity along $x=$ $x_{i}(t)$ still diminishes exponentially for $t \in\left(T_{0}, T^{*}\right]$, though it might be at a slightly changed rate. This implies that $v \in\left[v_{0}, v^{-}\right]$on $x=x_{1}(t)$ and $v \in\left[v_{0}, v^{+}\right]$on 
$x=x_{2}(t)$ for $x \in\left(T_{0}, T^{*}\right]$. Thus, for any point $\left(t_{1}, x_{2}\left(t_{1}\right)\right)$ with $t_{1} \in\left(T_{0}, T^{*}\right]$, the corresponding forward characteristic $x_{2}\left(\tau ; t_{1}, x_{2}\left(t_{1}\right)\right)$ will meet $x=x_{1}(t)$ at $\left(t_{2}, x_{1}\left(t_{2}\right)\right)$ with $t_{2} \leq T_{0}$ if $\delta$ is small enough. Therefore,

$$
0<v^{-1 / 2} s_{x}+v^{1 / 2}<\max \left\{N\left(T^{*}\right),\left(v^{-}\right)^{1 / 2}\right\}
$$

holds at $\left(t_{1}, x_{2}\left(t_{1}\right)\right)$ where $N\left(T^{*}\right) \leq \max \left\{\left(v^{+}\right)^{1 / 2},\left(v^{-}\right)^{1 / 2}\right\}$, due to the same argument as in Lemma 2.5. Thus,

$$
0<v^{-1 / 2} s_{x}+v^{1 / 2}<\left(v^{+}\right)^{1 / 2}
$$

holds at $\left(t_{1}, x_{2}\left(t_{1}\right)\right)$. In view of (2.34), Lemma 2.3, and the fact that $v \in\left[v_{0}, v^{+}\right]$ on $x_{2}(t)$ for $t \in\left(T_{0}, T^{*}\right]$, it follows under Condition A that the left half of (2.24) is true for any $\left(t, x_{2}(t)\right)$ with $t \in\left(T_{0}, T^{*}\right]$. Namely,

$$
\begin{aligned}
v^{-1 / 2} r_{x}+v^{1 / 2} & =\sqrt{v_{+}}\left[1-\left(\frac{\sqrt{v_{+}}-\sqrt{v}}{\sqrt{v_{+}}+\sqrt{v}}\right)^{3}\right]-\left(\frac{\sqrt{v_{+}}-\sqrt{v}}{\sqrt{v_{+}}+\sqrt{v}}\right)^{3}\left(v^{-1 / 2} s_{x}+v^{1 / 2}\right) \\
& >\sqrt{v^{+}}\left[1-2\left(\frac{\sqrt{v_{+}}-\sqrt{v}}{\sqrt{v^{+}}+\sqrt{v}}\right)^{3}\right]>0 .
\end{aligned}
$$

Similarly, for any point $\left(\bar{t}_{1}, x_{1}\left(\bar{t}_{1}\right)\right)$ with $\bar{t} \in\left(T_{0}, T^{*}\right]$, the corresponding backward characteristic $x_{1}\left(\tau ; t_{1}, x_{1}\left(t_{1}\right)\right)$ will meet $x=x_{2}(t)$ at $\left(\bar{t}_{2}, x_{2}\left(\bar{t}_{2}\right)\right)$ with $\bar{t}_{2} \leq T_{0}$, and

$$
0<v^{-1 / 2} r_{x}+v^{1 / 2}<\left(v^{+}\right)^{1 / 2}
$$

holds at $\left(\bar{t}_{1}, x_{1}\left(\bar{t}_{1}\right)\right)$. In view of (2.35), Lemma 2.3, and the fact that $v \in\left[v_{0}, v^{-}\right]$ on $x_{1}(t)$ for $t \in\left(T_{0}, T^{*}\right]$, it follows under Condition A that

$$
\begin{aligned}
v^{-1 / 2} s_{x}+v^{1 / 2} & =\sqrt{v_{-}}\left[1-\left(\frac{\sqrt{v_{-}}-\sqrt{v}}{\sqrt{v_{-}}+\sqrt{v}}\right)^{3}\right]-\left(\frac{\sqrt{v_{-}}-\sqrt{v}}{\sqrt{v_{-}}+\sqrt{v}}\right)^{3} \cdot\left(v^{-1 / 2} r_{x}+v^{1 / 2}\right) \\
& >\sqrt{v_{-}}-\left(\sqrt{v_{-}}+\sqrt{v_{+}}\right)\left(\frac{\sqrt{v_{-}}-\sqrt{v}}{\sqrt{v_{-}}+\sqrt{v}}\right)^{3} \\
& \geq \sqrt{v_{-}}-\left(\sqrt{v_{-}}+\sqrt{v_{+}}\right) \cdot\left(\frac{\sqrt{v_{-}}-\sqrt{v_{0}}}{\sqrt{v_{-}}+\sqrt{v_{0}}}\right)^{3} \\
& =\sqrt{v^{-}}-\left(\sqrt{v^{-}}+\sqrt{v^{+}}\right) \cdot\left(\frac{\sqrt{v^{-}}-\sqrt{v_{0}}}{\sqrt{v^{-}}+\sqrt{v_{0}}}\right)^{3}
\end{aligned}
$$

at any $\left(t, x_{1}(t)\right)$ with $t \in\left(T_{0}, T^{*}\right]$.

Define $F(\xi)=\xi\left[\left(\frac{\xi+1}{\xi-1}\right)^{3}-1\right]$. It is easy to see that

$$
F^{\prime}(\xi)=-\frac{18 \xi^{2}+4 \xi+2}{(\xi-1)^{4}}<0
$$


Due to $\sqrt{v^{-}} \leq \sqrt{v^{+}}<\frac{2^{1 / 3}+1}{2^{1 / 3}-1} \sqrt{v_{0}}$ and $F\left(\xi_{1}\right)=\xi_{1}$ for $\xi_{1}=\frac{2^{1 / 3}+1}{2^{1 / 3}-1}$, it follows that

$$
\frac{\sqrt{v^{-}}}{\sqrt{v_{0}}} \cdot\left[\left(\frac{\sqrt{v^{-}}+\sqrt{v_{0}}}{\sqrt{v^{-}}-\sqrt{v_{0}}}\right)^{3}-1\right]>\frac{2^{1 / 3}+1}{2^{1 / 3}-1} \text {. }
$$

Therefore,

$$
\frac{\sqrt{v^{+}}}{\sqrt{v_{0}}}<\frac{2^{1 / 3}+1}{2^{1 / 3}-1}<\frac{\sqrt{v^{-}}}{\sqrt{v_{0}}}\left[\left(\frac{\sqrt{v^{-}}+\sqrt{v_{0}}}{\sqrt{v^{-}}-\sqrt{v_{0}}}\right)^{3}-1\right] .
$$

It turns out then that

$$
v^{-1 / 2} s_{x}+v^{1 / 2}>0
$$

at $\left(t, x_{1}(t)\right)$ with $t \in\left(T_{0}, T^{*}\right]$, namely, the left half of (2.24) is true for any $\left(t, x_{1}(t)\right)$ with $t \in\left(T_{0}, T^{*}\right]$. With the help of (2.35) and (2.34), it is not difficult to show that the right halves of (2.22) and (2.24) are true for $t \in\left(T_{0}, T^{*}\right]$. It ends up then that

$$
\begin{array}{ll}
0<v^{-1 / 2} s_{x}+v^{1 / 2}<\left(v^{-}\right)^{1 / 2} & \text { along } x=x_{1}(t) \text { for } t \in\left[0, T^{*}\right], \\
0<v^{-1 / 2} r_{x}+v^{1 / 2}<\left(v^{+}\right)^{1 / 2} & \text { along } x=x_{2}(t) \text { for } t \in\left[0, T^{*}\right] .
\end{array}
$$

This with Lemma 2.6 together finishes Lemma 2.8. Namely, the constants $A_{i}$ ( $i=$ $1,2,3,4)$ in $\left(\mathrm{H}_{2}\right)$ can be reserved for $t \in\left[0, T^{*}\right]$. In fact, along $x=x_{2}(t)$,

$$
\begin{aligned}
& -\frac{d\left(\sqrt{v_{+}}-\sqrt{v}\right)}{\left(\sqrt{v_{+}}-\sqrt{v}\right) d t}=\frac{\sqrt{v v_{+}}}{\left(\sqrt{v_{+}}+\sqrt{v}\right)^{2}}+\frac{\sqrt{v}}{\left(\sqrt{v_{+}}+\sqrt{v}\right)^{2}}\left(v^{-1 / 2} s_{x}+v^{1 / 2}\right) \leq \frac{2 \sqrt{v v^{+}}}{\left(\sqrt{v^{+}}+\sqrt{v}\right)^{2}}<\frac{1}{2}, \\
& -\frac{d\left(\sqrt{v_{+}}-\sqrt{v}\right)}{\left(\sqrt{v_{+}}-\sqrt{v}\right) d t}>\frac{\sqrt{v v_{+}}}{\left(\sqrt{v_{+}}+\sqrt{v}\right)^{2}}>A_{2}
\end{aligned}
$$

for a certain positive constant $A_{2}$ that only depends on $v^{+}$and $v_{0}$-the lower bound of $v$ on $x=x_{2}(t)$. Similarly, along $x=x_{1}(t)$,

$$
-\frac{d\left(\sqrt{v_{-}}-\sqrt{v}\right)}{\left(\sqrt{v_{-}}-\sqrt{v}\right) d t} \in\left[A_{4}, \frac{1}{2}\right]
$$

where $A_{4}$ only depends on $v^{-}$and $v_{0}$-the lower bound of $v$ on $x=x_{1}(t)$.

Thus, Lemma 2.8 is proved.

Theorem 2.1 follows then from the above lemmas and local existence results.

3. Perturbed Riemann problem. We consider certain perturbed initial data (1.3) in this section, namely,

$$
\left(u_{0}(x), v_{0}(x)\right)= \begin{cases}\left(u_{-}(x), v_{-}(x)\right), & x<0, \\ \left(u_{+}(x), v_{+}(x)\right), & x>0,\end{cases}
$$

or in $(r, s)$ variables,

$$
\left(r_{0}(x), s_{0}(x)\right)= \begin{cases}\left(r_{-}(x), s_{-}(x)\right), & x<0, \\ \left(r_{+}(x), s_{+}(x)\right), & x>0,\end{cases}
$$


where the functions $u_{\mp}(x)$ and $v_{\mp}(x)$ are smooth, with

$$
\lim _{x \rightarrow 0_{\mp}}\left(u_{\mp}(x), v_{\mp}(x)\right)=\left(u^{\mp}, v^{\mp}\right) \quad\left(\text { or } \lim _{x \rightarrow 0_{\mp}}\left(r_{\mp}(x), s_{\mp}(x)\right)=\left(r^{\mp}, s^{\mp}\right)\right)
$$

such that $\left(u^{-}, v^{-}\right)$and $\left(u^{+}, v^{+}\right)$(or $\left(r^{-}, s^{-}\right)$and $\left.\left(r^{+}, s^{+}\right)\right)$satisfy Condition (2.15).

Without loss of generality, we assume that

$$
0<v_{ \pm}(x)<1, \quad u_{ \pm}(x)>0 .
$$

We will construct the global discontinuous solution for (1.1), (3.1) under the following restrictions on the perturbation in (3.1). Denote

$$
\begin{aligned}
& \underline{q^{-}}=\min \left\{\inf _{x<0}\left(\frac{1}{\sqrt{v_{-}(x)}} \cdot r_{-}^{\prime}(x)+\sqrt{v_{-}(x)}\right),\right. \\
& \left.\inf _{x<0}\left(\frac{1}{\sqrt{v_{-}(x)}} \cdot s_{-}^{\prime}(x)+\sqrt{v_{-}(x)}\right), \inf _{x<0} \sqrt{v_{-}(x)}\right\} \\
& \overline{q^{-}}=\max \left\{\sup _{x<0}\left(\frac{1}{\sqrt{v_{-}(x)}} \cdot r_{-}^{\prime}(x)+\sqrt{v_{-}(x)}\right)\right. \text {, } \\
& \left.\sup _{x<0}\left(\frac{1}{\sqrt{v_{-}(x)}} \cdot s_{-}^{\prime}(x)+\sqrt{v_{-}(x)}\right), \sup _{x<0} \sqrt{v_{-}(x)}\right\} \\
& \underline{q^{+}}=\min \left\{\inf _{x>0}\left(\frac{1}{\sqrt{v_{+}(x)}} \cdot r_{+}^{\prime}(x)+\sqrt{v_{+}(x)}\right)\right. \text {, } \\
& \left.\inf _{x>0}\left(\frac{1}{\sqrt{v_{+}(x)}} \cdot s_{+}^{\prime}(x)+\sqrt{v_{+}(x)}\right), \inf _{x>0} \sqrt{v_{+}(x)}\right\} \\
& \overline{q^{+}}=\max \left\{\sup _{x>0}\left(\frac{1}{\sqrt{v_{+}(x)}} \cdot r_{+}^{\prime}(x)+\sqrt{v_{+}(x)}\right)\right. \text {, } \\
& \left.\sup _{x>0}\left(\frac{1}{\sqrt{v_{+}(x)}} \cdot s_{+}^{\prime}(x)+\sqrt{v_{+}(x)}\right), \sup _{x>0} \sqrt{v_{+}(x)}\right\} .
\end{aligned}
$$

Condition B. For some positive constants $\varepsilon, \tilde{\varepsilon}$, it holds that

1.

$$
\begin{aligned}
& \sqrt{v^{-}} \tilde{\varepsilon}<\sqrt{v_{0}}<\sqrt{v^{-}}(1-\tilde{\varepsilon}) \leq q^{-} \leq \overline{q^{-}} \leq \sqrt{v^{-}}(1+\tilde{\varepsilon}) \\
& \sqrt{v^{+}} \varepsilon<\sqrt{v_{0}}<\sqrt{v^{+}}(1-\varepsilon) \leq \underline{q}^{+} \leq \overline{q^{+}} \leq \sqrt{v^{+}}(1+\varepsilon) .
\end{aligned}
$$

Define $\xi_{0}^{-}$and $\xi_{0}^{+}$by $\sqrt{v^{-}}=\xi_{0}^{-} \sqrt{v^{0}}$ and $\sqrt{v^{+}}=\xi_{0}^{+} \sqrt{v_{0}}$, respectively, where $\xi_{0}^{+}>1, \xi_{0}^{-}>1$. Moreover, the following inequalities are satisfied: 
2. $-\left[\frac{\xi_{0}^{-}-1}{\xi_{0}^{-}(1-2 \tilde{\varepsilon})+1}\right]^{3} \cdot\left[\max \left\{\xi_{0}^{+}(1+\varepsilon), \xi_{0}^{-}(1+\tilde{\varepsilon})\right\}+\xi_{0}^{-}(1+\tilde{\varepsilon})\right]+\xi_{0}^{-}(1-\tilde{\varepsilon})>0$,

3. $-\left[\frac{\xi_{0}^{+}-1}{\xi_{0}^{+}(1-2 \varepsilon)+1}\right]^{3} \cdot\left[\max \left\{\xi_{0}^{+}(1+\varepsilon), \xi_{0}^{-}(1+\tilde{\varepsilon})\right\}+\xi_{0}^{+}(1+\varepsilon)\right]+\xi_{0}^{+}(1-\tilde{\varepsilon})>0$.

4. For any $\xi \in\left[1, \frac{\sqrt{v^{-}}(1+\tilde{\varepsilon})}{\sqrt{v_{0}}-\sqrt{v^{-}} \cdot \tilde{\varepsilon}}\right]$,

$$
\frac{1}{2}+\frac{\left(\xi^{3}+\xi^{2}+3 \xi-1\right)}{4(\xi+1)^{2}} \cdot \frac{(1-\tilde{\varepsilon})}{(1+\tilde{\varepsilon})}-\frac{(\xi+1)}{4} \frac{(1+\tilde{\varepsilon})}{(1-\tilde{\varepsilon})}>0
$$

holds.

5. For any $\xi \in\left[1, \frac{\sqrt{v^{+}}(1+\varepsilon)}{\sqrt{v_{0}}-\sqrt{v^{+}} \tilde{\varepsilon}}\right]$,

$$
\frac{1}{2}+\frac{\left(\xi^{3}+\xi^{2}+3 \xi-1\right)}{4(\xi+1)^{2}} \cdot \frac{(1-\varepsilon)}{(1+\varepsilon)}-\frac{(\xi+1)}{4} \cdot \frac{(1+\varepsilon)}{(1-\varepsilon)}>0
$$

holds.

6. Denote $\eta_{0}^{ \pm}=\left(\frac{\xi_{1}^{ \pm}-1}{\xi_{0}^{ \pm}+1}\right)^{3}, K_{1}\left(\xi_{1}, \xi_{2}, \eta_{1}, \eta_{2}\right)=\xi_{1}-\eta_{1}\left(\xi_{1}+\xi_{2}\right)+\eta_{1} \eta_{2} \xi_{2}, K_{2}\left(\xi_{1}, \xi_{2}\right.$, $\left.\eta_{1}, \eta_{2}, \varepsilon_{1}, \varepsilon_{2}\right)=\varepsilon_{1} \xi_{1}\left(1+\eta_{1}\right)+\varepsilon_{2} \eta_{1} \xi_{2}\left(1+\eta_{2}\right)$. Then

$$
\begin{aligned}
& K_{1}\left(\xi_{0}^{-}, \xi_{0}^{+}, \eta_{0}^{-}, \eta_{0}^{+}\right)-K_{2}\left(\xi_{0}^{-}, \xi_{0}^{+}, \eta_{0}^{-}, \eta_{0}^{+}, \tilde{\varepsilon}, \varepsilon\right) \geq 1-\eta_{0}^{+} \eta_{0}^{-}, \\
& K_{1}\left(\xi_{0}^{-}, \xi_{0}^{+}, \eta_{0}^{-}, \eta_{0}^{+}\right)+K_{2}\left(\xi_{0}^{-}, \xi_{0}^{+}, \eta_{0}^{-}, \eta_{0}^{+}, \tilde{\varepsilon}, \varepsilon\right) \leq\left(1-\eta_{0}^{+} \eta_{0}^{-}\right) \max \left\{\xi_{0}^{-}, \xi_{0}^{+}\right\}, \\
& K_{1}\left(\xi_{0}^{+}, \xi_{0}^{-}, \eta_{0}^{+}, \eta_{0}^{-}\right)-K_{2}\left(\xi_{0}^{+}, \xi_{0}^{-}, \eta_{0}^{+}, \eta_{0}^{-}, \varepsilon, \tilde{\varepsilon}\right) \geq 1-\eta_{0}^{+} \eta_{0}^{-}, \\
& K_{1}\left(\xi_{0}^{+}, \xi_{0}^{-}, \eta_{0}^{+}, \eta_{0}^{-}\right)+K_{2}\left(\xi_{0}^{+}, \xi_{0}^{-}, \eta_{0}^{+}, \eta_{0}^{-}, \varepsilon, \tilde{\varepsilon}\right) \leq\left(1-\eta_{0}^{+} \eta_{0}^{-}\right) \max \left\{\xi_{0}^{-}, \xi_{0}^{+}\right\}
\end{aligned}
$$

hold.

Remark 3.1. When $\tilde{\varepsilon}$ and $\varepsilon$ tend to zero, the inequalities $B_{4}, B_{5}$, and $B_{6}$ hold automatically, $B_{1}$ holds as well if $r_{ \pm}^{\prime}(x) \equiv 0$ and $s_{\mp}^{\prime}(x) \equiv 0$, while $B_{2}$ and $B_{3}$ hold under Condition A. This means that Condition B is a direct generalization of Condition A. Moreover, it can be claimed that for any fixed positive number $b$, $b<\frac{2^{1 / 3}+1}{2^{1 / 3}-1}$, there exist positive constants $\varepsilon, \tilde{\varepsilon}$, and $\varepsilon_{0}$ such that all of the above inequalities in Condition B hold for this pair of $(\varepsilon, \tilde{\varepsilon})$ if

$$
\frac{\max \left\{\sqrt{v^{-}}, \sqrt{v^{+}}\right\}}{\sqrt{v_{0}}} \leq b
$$

and

$$
\operatorname{osc} v_{\mp}(x)+\left|r_{\mp}^{\prime}(x)\right|+\left|s_{\mp}^{\prime}(x)\right| \leq \varepsilon_{0} \quad \text { (for } x \leq 0 \text { or } x \geq 0 \text { respectively). }
$$

THEOREM 3.2. Suppose that $\left(r_{\mp}(x), s_{\mp}(x)\right)$ are $C^{1}$ functions with bounded $C^{1}$ norm such that $\underline{q}^{\mp}>0$. Then there exist globally-defined classical solutions $\left(r_{+}(t, x)\right.$, $\left.s_{+}(t, x)\right) \in C^{1}$ and $\left(r_{-}(t, x), s_{-}(t, x)\right) \in C^{1}$ in the regions $\widehat{R}_{+}$and $\widehat{R}_{-}$, respec- 
500

L. HSIAO AND S. Q. TANG

tively, such that

$$
\begin{gathered}
\operatorname{In} \hat{R}_{-}: s_{*}^{-} \leq s \leq \sup _{x \leq 0} s_{-}(x), \\
v^{1 / 2} \in\left[\underline{q}^{-}, \overline{q^{-}}\right], \quad\left(v^{-1 / 2} r_{x}+v^{1 / 2}\right) \in\left[\underline{q}^{-}, \overline{q^{-}}\right], \quad\left(v^{-1 / 2} s_{x}+v^{1 / 2}\right) \in\left[\underline{q}^{-}, \overline{q^{-}}\right], \\
\operatorname{In} \hat{R}^{+}: s_{*}^{+} \leq s \leq \sup _{x \geq 0} s_{+}(x), \\
v^{1 / 2} \in\left[\underline{q}^{+}, \overline{q^{+}}\right], \\
\left(v^{-1 / 2} r_{x}+v^{1 / 2}\right)
\end{gathered}
$$

where the subscript with the solution $(r, s)$ is omitted, and $s_{*}^{-}$and $s_{*}^{+}$are defined by $s_{*}^{-}=-\ln \left(\sup _{x \leq 0} v_{-}(x)\right)$ and $s_{*}^{+}=-\ln \left(\sup _{x \geq 0} v_{+}(x)\right)$, respectively.

Just as in Sec. 2 , the discontinuous initial-value problem (1.1), (3.1) admits a unique discontinuous solution at least on a local domain $R(\delta)$ in a class of piecewisesmooth functions and this solution contains only a backward shock $x=x_{1}(t)$ and a forward shock $x=x_{2}(t)$ passing through the origin. Moreover, the solutions on the left side of $x=x_{1}(t)$ and on the right side of $x=x_{2}(t)$ are furnished by $\left(r_{-}(t, x), s_{-}(t, x)\right)$ and $\left(r_{+}(t, x), s_{+}(t, x)\right)$, respectively, and one is required to solve the same free boundary problem (FBP) on the angular domain $R$ as in Sec. 2 in order to construct the globally-defined discontinuous solution for (1.1), (3.1) that contains only two shocks.

LEMmA 3.3. Suppose that the classical solution of (1.1), (2.16), (2.17) exists in $R(T)$. Then

$$
s_{x}=\frac{4 v \sqrt{v_{-}}\left(\sqrt{v_{-}}-\sqrt{v}\right)}{\sqrt{v_{-}}+\sqrt{v}}-\left(\frac{\sqrt{v_{-}}-\sqrt{v}}{\sqrt{v_{-}}+\sqrt{v}}\right)^{3}\left(r_{x}+\sqrt{\frac{v}{v_{-}}}\left(r_{-}\right)_{x}\right)+\sqrt{\frac{v}{v_{-}}}\left(s_{-}\right)_{x}
$$

or

$$
\begin{aligned}
\frac{s_{x}}{\sqrt{v}}+\sqrt{v}=- & \left(\frac{\sqrt{v_{-}}-\sqrt{v}}{\sqrt{v_{-}}+\sqrt{v}}\right)^{3} \cdot\left[\left(\frac{r_{x}}{\sqrt{v}}+\sqrt{v}\right)+\left(\frac{\left(r_{-}\right)_{x}}{\sqrt{v_{-}}}+\sqrt{v_{-}}\right)\right] \\
+ & \left(\frac{\left(s_{-}\right)_{x}}{\left.\sqrt{v_{-}}+\sqrt{v_{-}}\right),}\right. \\
-\frac{d\left(\sqrt{v_{-}}-\sqrt{v}\right)}{\left(\sqrt{v_{-}}-\sqrt{v}\right) d t}= & \frac{1}{2}+\frac{\sqrt{v}}{\left(\sqrt{v_{-}}+\sqrt{v}\right)^{2}} \cdot\left(\frac{r_{x}}{\sqrt{v}}+\sqrt{v}\right) \\
& +\frac{v_{-} \sqrt{v_{-}}+v_{-} \sqrt{v}+3 \sqrt{v_{-}} v-v \sqrt{v}}{4 \sqrt{v v_{-}}\left(\sqrt{v_{-}}+\sqrt{v}\right)^{2}} \cdot\left(\frac{\left(r_{-}\right)_{x}}{\sqrt{v_{-}}}+\sqrt{v_{-}}\right) \\
& -\frac{\sqrt{v_{-}}+\sqrt{v}}{4 \sqrt{v_{-} v}}\left(\frac{\left(s_{-}\right)_{x}}{\sqrt{v_{-}}}+\sqrt{v_{-}}\right)
\end{aligned}
$$

hold along $x=x_{1}(t)$ while

$$
r_{x}=\frac{4 v \sqrt{v_{+}}\left(\sqrt{v_{+}}-\sqrt{v}\right)}{\left(\sqrt{v_{+}}+\sqrt{v}\right)^{2}}-\left(\frac{\sqrt{v_{+}}-\sqrt{v}}{\sqrt{v_{+}}+\sqrt{v}}\right)^{3}\left(s_{x}+\sqrt{\frac{v}{v_{+}}}\left(s_{+}\right)_{x}\right)+\sqrt{\frac{v}{v_{+}}}\left(r_{+}\right)_{x}
$$


or

$$
\begin{aligned}
\frac{r_{x}}{\sqrt{v}}+\sqrt{v}=-\left(\frac{\sqrt{v_{+}}-\sqrt{v}}{\sqrt{v_{+}}}+\sqrt{v}\right)^{3} \cdot\left[\left(\frac{s_{x}}{\sqrt{v}}+\sqrt{v}\right)+\left(\frac{\left(s_{+}\right)_{x}}{\sqrt{v_{+}}}+\sqrt{v_{+}}\right)\right]+\left(\frac{\left(r_{+}\right)_{x}}{\sqrt{v_{+}}+\sqrt{v_{+}}}\right) \\
-\frac{d\left(\sqrt{v_{+}}-\sqrt{v}\right)}{\left(\sqrt{v_{+}}-\sqrt{v}\right) d t}=\frac{1}{2}+\frac{\sqrt{v}}{\left(\sqrt{v_{+}}+\sqrt{v}\right)^{2}} \cdot\left(\frac{s_{x}}{\sqrt{v}}+\sqrt{v}\right) \\
+\frac{v_{+} \sqrt{v_{+}}+v_{+} \sqrt{v}+3 \sqrt{v_{+}} v-v \sqrt{v}}{4 \sqrt{v v_{+}}\left(\sqrt{v_{+}}+\sqrt{v}\right)^{2}} \cdot\left(\frac{\left(s_{+}\right)_{x}}{\sqrt{v_{+}}}+\sqrt{v_{+}}\right) \\
-\frac{\sqrt{v_{+}}+\sqrt{v}}{4 \sqrt{v_{+} v}}\left(\frac{\left(r_{+}\right)_{x}}{\sqrt{v_{+}}}+\sqrt{v_{+}}\right)
\end{aligned}
$$

hold along $x=x_{2}(t)$ where

$$
\begin{array}{lll}
v_{-}=v_{-}\left(t, x_{1}(t)\right), & r_{-}=r_{-}\left(t, x_{1}(t)\right), & s_{-}=s_{-}\left(t, x_{1}(t)\right), \\
v_{+}=v_{+}\left(t, x_{2}(t)\right), & r_{+}=r_{+}\left(t, x_{2}(t)\right), & s_{+}=s_{+}\left(t, x_{2}(t)\right), \text { etc. }
\end{array}
$$

TheOREM 3.4. Under Condition B, the perturbed free boundary problem admits a global classical solution $(r, s) \in C^{1}$ in $R$ with $x_{i}(t) \in C^{2}(i=1,2)$ on which (2.18) holds. Furthermore, this solution possesses the following properties. For any $(t, x) \in R$,

$$
\begin{aligned}
s_{*} & \leq s \leq \sup _{x \leq 0} s_{-}(x), \quad-\left(\sup _{x \leq 0} \underline{s}(x)\right) \leq r \leq r^{*}, \quad \sqrt{v} \leq \max \left\{\overline{q^{-}}, \overline{q^{+}}\right\} \\
& \min \left\{\min _{\substack{x_{2}(\tau ; t, x) \\
\alpha \leq \tau \leq t}} v^{1 / 2},\left(v^{-1 / 2} s_{x}+v^{1 / 2}\right)\left(\alpha, x_{1}(\alpha)\right)\right\}, \\
& \leq v^{-1 / 2} s_{x}+v^{1 / 2} \leq \max \left\{\max _{\substack{x_{2}(\tau ; t, x) \\
\alpha \leq \tau \leq t}} v^{1 / 2},\left(v^{-1 / 2} s_{x}+v^{1 / 2}\right)\left(\alpha, x_{1}(\alpha)\right)\right\},
\end{aligned}
$$

$$
\begin{aligned}
\min \left\{\min _{\substack{x_{1}(\tau ; t, x) \\
\beta \leq \tau \leq t}} v^{1 / 2}\right. & \left.,\left(v^{-1 / 2} r_{x}+v^{1 / 2}\right)\left(\beta, x_{2}(\beta)\right)\right\}, \\
& \leq v^{-1 / 2} r_{x}+v^{1 / 2} \leq \max \left\{\max _{\substack{x_{1}(\tau ; t, x) \\
\beta \leq \tau \leq t}} v^{1 / 2},\left(v^{-1 / 2} r_{x}+v^{1 / 2}\right)\left(\beta, x_{2}(\beta)\right)\right\},
\end{aligned}
$$

where the notation of $x_{i}(\tau ; t, x),\left(\beta, x_{2}(\beta)\right)$, and $\left(\alpha, x_{1}(\alpha)\right)$ is defined in the same way as in Theorem $2.1, s^{*}$ is defined as the $s$-value at the interaction point of $r+s=0$ and $v=\left(\max \left\{\overline{q^{+}}, \overline{q^{-}}\right\}\right)^{2}$, namely, $s_{*}=-2 \ln \left(\max \left\{\overline{q^{+}}, \overline{q^{-}}\right\}\right), r^{*}$ is defined as the $r$-value at the intersection point of $s=\sup _{x \leq 0} s_{-}(x)$ and the curve $r_{R}-r=h_{2}\left(s_{R}-s\right)$ given in (2.12), where $s_{R}=\sup _{x \geq 0} s_{+}(x), r_{R}=s_{R}+4 \ln \overline{q^{+}}$, namely, $r^{*}=\sup _{x \geq 0} s_{+}(x)+4 \ln \overline{q^{+}}-h_{2}\left(\sup _{x \geq 0} s_{+}(x)-\sup _{x \leq 0} s_{-}(x)\right)$. Moreover, both shocks diminish exponentially, namely, there exist positive constants $A_{i}, i=1, \ldots, 4$ such that

$$
\left(\sqrt{v^{-}}-\sqrt{v_{0}}\right) e^{-A_{3} t} \leq \sqrt{v_{-}}-\sqrt{v} \leq\left(\sqrt{v^{-}}-\sqrt{v_{0}}\right) e^{-A_{4} t}
$$


along $x=x_{1}(t)$;

$$
\left(\sqrt{v^{+}}-\sqrt{v_{0}}\right) e^{-A_{1}(t)} \leq \sqrt{v_{+}}-\sqrt{v} \leq\left(\sqrt{v^{+}}-\sqrt{v_{0}}\right) e^{-A_{2} t}
$$

along $x=x_{2}(t)$.

Proof. Let us denote the following statement by $\mathrm{H}_{2}^{\prime}$. $\left(\mathrm{H}_{2}^{\prime}\right)$ Along $x=x_{1}(t)$, it holds that

$$
0<\frac{s_{x}}{\sqrt{v}}+\sqrt{v}<\sqrt{v^{-}}(1+\tilde{\varepsilon})
$$

and

$$
\frac{-1}{\left(\sqrt{v_{-}}-\sqrt{v}\right)} \frac{d\left(\sqrt{v_{-}}-\sqrt{v}\right)}{d t} \in\left[A_{4}, A_{3}\right] .
$$

Along $x=x_{2}(t)$, it holds that

$$
0<\frac{r_{x}}{\sqrt{v}}+\sqrt{v}<\sqrt{v^{+}}(1+\varepsilon)
$$

and

$$
-\frac{1}{\left(\sqrt{v_{+}}-\sqrt{v}\right)} \frac{d\left(\sqrt{v_{+}}-\sqrt{v}\right)}{d t} \in\left[A_{2}, A_{1}\right],
$$

where $A_{i}(i=1,2,3,4)$ are positive constants.

We prove Theorem 3.4 next by the same framework as used in Sec. 2 .

First, it can be shown by the same argument as in Lemma 2.5 that under Condition $\mathrm{B}_{1}$, the classical solution defined in $R(T)$ satisfies the estimates cited in (3.11)-(3.14) if $\left(\mathrm{H}_{1}\right)$ and $\left(\mathrm{H}_{2}^{\prime}\right)$ hold in $R(T)$.

Also similar to the argument of Sec. 2 one can prove that $\left(\mathrm{H}_{1}\right)$ and $\left(\mathrm{H}_{2}^{\prime}\right)$ hold locally in $t$.

Denote $R=r_{x} / \sqrt{v}+\sqrt{v}, S=s_{x} / \sqrt{v}+\sqrt{v}$,

$$
R^{\mp}=\frac{\left(r_{\mp}\right)_{x}}{\sqrt{v_{\mp}}}+\sqrt{v_{\mp}}, \quad S^{\mp}=\frac{\left(s_{\mp}\right)_{x}}{\sqrt{v_{\mp}}}+\sqrt{v_{\mp}},
$$

where $r_{-}=r_{-}(t, x), r_{+}=r_{+}(t, x)$, etc. It follows, from Lemma 3.3, that

$$
\begin{aligned}
& S=-\eta_{0}^{-}\left(R+R^{-}\right)+S^{-}, \\
& R=-\eta_{0}^{+}\left(S+S^{+}\right)+R^{+} .
\end{aligned}
$$

Thus,

$$
S=\frac{S^{-}-\eta_{0}^{-}\left(R^{-}+R^{+}\right)+\eta_{0}^{-} \eta_{0}^{+} S^{+}}{1-\eta_{0}^{-} \eta_{0}^{+}},
$$

which implies that (3.15) holds as $t$ is small enough due to Condition $\mathrm{B}_{6}$, and similarly for (3.17). The other part of showing $\left(\mathrm{H}_{1}\right)$ and $\left(\mathrm{H}_{2}^{\prime}\right)$ locally in $t$ can be obtained by an argument similar to that in Sec. 2 .

We prove next that the solution constructed by extension reserves $\left(\mathrm{H}_{1}\right)$ and $\left(\mathrm{H}_{2}^{\prime}\right)$ in each step under Condition B. Namely, if $\left(\mathrm{H}_{1}\right)$ and $\left(\mathrm{H}_{2}^{\prime}\right)$ hold in $R\left(T_{0}\right)$ where the classical solution of the free boundary problem is defined, then the solution defined 
in $R\left(T_{0}+\delta\right)$ still satisfies $\left(\mathrm{H}_{1}\right)$ and $\left(\mathrm{H}_{2}^{\prime}\right)$, provided $\delta$ is small enough. For this purpose, we only need to discuss the difference from the unperturbed case in Sec. 2.

In view of (3.7),

$$
\frac{s_{x}}{\sqrt{v}}+\sqrt{v}=-\left(\frac{\sqrt{v_{-}}-\sqrt{v}}{\sqrt{v_{-}}+\sqrt{v}}\right)^{3} \cdot\left[\left(\frac{r_{x}}{\sqrt{v}}+\sqrt{v}\right)+\left(\frac{\left(r_{-}\right)_{x}}{\sqrt{v_{-}}}+\sqrt{v_{-}}\right)\right]+\left(\frac{\left(s_{-}\right)_{x}}{\sqrt{v_{-}}}+\sqrt{v_{-}}\right)
$$

holds on $x=x_{1}(t)$. By the same argument as in Lemma 2.8, it can be shown that

$$
0<\frac{r_{x}}{\sqrt{v}}+\sqrt{v}<\max \left\{\sqrt{v^{+}}(1+\varepsilon), \sqrt{v^{-}}(1+\tilde{\varepsilon})\right\}
$$

and

$$
\sqrt{v_{-}}-\sqrt{v} \leq \sqrt{v^{-}}-\sqrt{v_{0}}=\sqrt{v_{0}}\left(\xi_{0}^{-}-1\right)
$$

hold on $x=x_{1}(t)$ for $t \in\left(T_{0}, T^{*}\right]$.

Denote $\xi=\sqrt{v_{-}} / \sqrt{v}$. Due to (3.19) and (3.4), it follows that

$\frac{s_{x}}{\sqrt{v}}+\sqrt{v} \geq \sqrt{v_{0}}\left\{-\left(\frac{\xi-1}{\xi+1}\right)^{3} \cdot\left[\max \left\{\xi_{0}^{*}(1+\varepsilon), \xi_{0}^{-}(1+\tilde{\varepsilon})\right\}+\xi_{0}^{-}(1+\tilde{\varepsilon})\right]+\xi_{0}^{-}(1-\tilde{\xi})\right\}$.

It is obvious from (3.20) that

$$
\sqrt{v_{-}}+\sqrt{v} \geq 2 \sqrt{v_{-}}-\left(\sqrt{v^{-}}-\sqrt{v_{0}}\right) \geq \sqrt{v_{0}}\left[\xi_{0}^{-}(1-2 \tilde{\varepsilon})+1\right]
$$

which, combined with (3.20), shows that

$$
\frac{\xi-1}{\xi+1} \leq \frac{\xi_{0}^{-}-1}{\xi_{0}^{-}(1-2 \tilde{\varepsilon})+1} .
$$

Therefore, $s_{x} / \sqrt{v}+\sqrt{v}>0$ is true on $x=x_{1}(t)$ for $t \in\left[T_{0}, T^{*}\right]$ by Condition $\mathrm{B}_{2}$.

The other half of (3.15) can easily be obtained for $t \in\left(T_{0}, T^{*}\right]$. Similarly, it is true for (3.17) on $x=x_{2}(t), t \in\left(T_{0}, T^{*}\right]$, by Condition $\mathrm{B}_{3}$. We prove (3.16) next. In view of Lemma 3.3, Theorem 3.2, and (3.17),

$$
\begin{aligned}
\frac{-1}{\left(\sqrt{v_{-}}-\sqrt{v}\right)} \frac{d\left(\sqrt{v_{-}}-\sqrt{v}\right)}{d t}= & \frac{1}{2}+\frac{\sqrt{v}}{\left(\sqrt{v_{-}}+\sqrt{v}\right)^{2}} \cdot\left(\frac{r_{x}}{\sqrt{v}}+\sqrt{v}\right) \\
& +\frac{\left(v_{-} \sqrt{v_{-}}+v_{-} \sqrt{v}+3 \sqrt{v_{-}} v-v \sqrt{v}\right)}{4 \sqrt{v v_{-}}\left(\sqrt{v_{-}}+\sqrt{v}\right)^{2}} \cdot\left(\frac{\left(r_{-}\right)_{x}}{\sqrt{v_{-}}}+\sqrt{v_{-}}\right) \\
& -\frac{\left(\sqrt{v_{-}}+\sqrt{v}\right)}{4 \sqrt{v_{1} v}}\left(\frac{\left(s_{-}\right)_{x}}{\sqrt{v_{-}}}+\sqrt{v_{-}}\right) \\
\geq & \frac{1}{2}+\frac{\left(\xi^{3}+\xi^{2}+3 \xi-1\right)}{4(\xi+1)^{2}} \frac{(2-\tilde{\varepsilon})}{(1+\tilde{\varepsilon})}-\frac{(\xi+1)}{4} \cdot \frac{(1+\tilde{\varepsilon})}{(1-\tilde{\varepsilon})}
\end{aligned}
$$

holds on $x=x_{1}(t)$.

Due to (3.20), (3.4), and Condition $B_{1}$, it is known that

$$
\xi \leq \frac{\sqrt{v^{-}}(1+\tilde{\varepsilon})}{\sqrt{v_{0}}-\sqrt{v^{-}} \tilde{\varepsilon}} \text {. }
$$


Therefore, it follows that

$$
\frac{-1}{\left(\sqrt{v_{-}}-\sqrt{v}\right)} \frac{d\left(\sqrt{v_{-}}-\sqrt{v}\right)}{d t} \geq A_{4}>0
$$

by Condition $\mathrm{B}_{4}$.

(3.18) can be obtained from Condition $\mathrm{B}_{5}$ similarly.

Theorem 3.4 is then proved.

4. The case when $\gamma$ is nearly one. The method used in Sec. 2 and Sec. 3 can be used to investigate the case when $1<\gamma<3$. However, the result will not be so good as previously if $\gamma$ is not very close to one. For instance, the condition $\inf _{R(T)} g>\sqrt{\frac{2(\gamma-1)}{\gamma+1}} \sup _{R(T)} g\left(g=\frac{4 \gamma^{1 / 4}}{3-\gamma} v^{(3-\gamma) / 4}\right)$ would make a much more strict restriction if $\gamma$ is not nearly 1 . We omit the details on the discussion that follows the same lines as in Sec. 2 and Sec. 3 to establish the result for the case when $\gamma$ is nearly one. Instead, we introduce a complete investigation for $1<\gamma<3$ in [HT] by different methods.

Acknowledgments. The first author would like to thank Professor R. LeVeque and Professor C. M. Dafermos for valuable discussions and their encouragement in investigating this problem.

\section{REFERENCES}

[CH] T. Chang and L. Hsiao, The Riemann Problem and Interaction of Waves in Gas Dynamics, Pitman Monographs and Surveys in Pure and Applied Mathematics 41, Longman, 1989

[DD] C. M. Dafermos and R. DiPerna, The Riemann problem for certain classes of hyperbolic systems of conservation laws, J. Diff. Eqs. 20, 90-114 (1976)

[DH] C. M. Dafermos and L. Hsiao, Hyperbolic systems of balance laws with inhomogeneity and dissipation, Indiana Univ. Math. J. 31, 471-491 (1982)

[DCL] X. X. Ding, G. Q. Chen, and P. Luo, Convergence of the fractional step Lax-Friedrichs scheme and Godunov scheme for the isentropic system of gas dynamics, Comm. Math. Phys. 121, 63-84 (1989)

[HL] L. Hsiao and Tai-ping Liu, Convergence to Nonlinear Diffusion Waves for Solutions of a System of Hyperbolic Conservation Laws with Damping, Comm. Math. Phys. 143, 599-605 (1992)

[HM] L. Hsiao and P. Marcati, Nonhomogeneous Quasilinear Hyperbolic Systems Arising in Chemical Engineering, Scuola Normale Superiore, Pisa, 1988, pp. 65-97

[HT] L. Hsiao and S. Q. Tang, Construction and qualitative behavior of solution of perturbed Riemann problem for the system of one-dimensional isentropic flow with damping (accepted by J. Diff. Eqs.)

[ $\left.\mathrm{LI}_{1}\right]$ Tai-ping Liu, Existence and uniqueness theorems for Riemann problems, Trans. Amer. Math. Soc. 213, 375-382 (1975)

[LI $]$ Tai-ping Liu, Quasilinear hyperbolic systems, Comm. Math. Phys. 68, 141-172 (1979)

[LT] M. Luskin and B. Temple, The existence of a global weak solution to the nonlinear waterhammer problem, Comm. Pure Appl. Math. 35, 697-735 (1982)

[LY] Ta-tsien Li and Wen-ci Yu, Boundary value problems for quasilinear hyperbolic systems, Duke University Mathematics Series V, 1985

[LZ] Ta-tsien $\mathrm{Li}$ and Yan-chun Zhao, Global perturbation of the Riemann problem for the system of one-dimensional isentropic flow, Lecture Notes in Mathematics 1306, Springer-Verlag, 1988, pp. 131-140

[SM] J. Smoller, Shock Waves and Reaction-Diffusion Equations, Springer-Verlag, 1980 
[TX $\mathrm{TX}_{1}$ S. Q. Tang and L. Xiao, Global perturbation of the Riemann problem for the system of compressible flow through porous media (I)

[TX $\mathrm{TX}_{2}$ S. Q. Tang and L. Xiao, Global perturbation of the Riemann problem for the system of compressible flow through porous media (II)

[YW] Lung-An Ying and Ching-Hua Wang, Global solutions of the Cauchy problem for a nonhomogeneous quasilinear hyperbolic system, Comm. Pure Appl. Math. 33, 579-597 (1980) 